\title{
Prise de position concernant les restrictions actuelles dans la pratique de la PMA en Suisse
}

Gabriel de Candollea,

Christian De Geyter ${ }^{b}$

a Dr, Président de la Société Suisse de Médecine de la Reproduction (SSMR)

b Prof. Dr, Président du registre national FIVNAT-CH

Depuis 2001, la PMA est régulée en Suisse par une législation contraignante qui, entre autres restrictions, limite le développement de plus de 3 ovocytes imprégnés et interdit toute cryoconservation d'embryons. De plus aucune aide financière n'est accordée par l'assurance maladie. Cet élément ajoute une pression importante sur les couples déjà physiquement et psychologiquement atteints par leur infertilité et les traitements qu'elle nécessite.

Malgré ces contraintes, le nombre de traitements augmente chaque année. Actuellement environ 8000 traitements de PMA (procréation médicalement assistée) sont conduits annuellement en Suisse. Le besoin est accentué par la tendance démographique des couples (hommes et femmes) à retarder l'âge de la première grossesse. Ce retard augmente la probabilité de maladies interférant avec la fertilité. Même si ces conditions ne nécessitent pas toujours le recours à la PMA, la FIV (fécondation in vitro) et la microinjection des spermatozoïdes sont actuellement les traitements reconnus pour l'infertilité féminine et masculine en Suisse et dans le monde. Dans certains pays, ces traitements sont à l'origine de 4,5\% des naissances. En Suisse, la proportion n'est que de $1,5 \%$, vraisemblablement en raison des restrictions légales et des contraintes financières sur les couples. Différentes études dans

\section{«Nous demandons une révision des restrictions en cours actuellement. Les modifications minimales suivantes sont demandées.»}

Correspondance: Dr Gabriel de Candolle 6 , rue de Candolle CH-1205 Genève Tél. 0227815380 divers pays ont démontré que le remboursement de la PMA représente entre 0,06 et $0,25 \%$ des dépenses de santé seulement, Ces coûts sont largement compensés par la participation des enfants nés après ces traitements à la prospérité commune durant leur vie.

La nécessité de replacer dans l'utérus tous les embryons obtenus lors d'un traitement vient de l'interdiction de congeler des embryons. Cette obligation conduit à transférer un plus grand nombre d'embryons que ce qui est actuellement considéré acceptable dans les pays qui ont une législation plus libé- rale. Cette pratique conduit à un plus grand nombre de grossesses multiples, surtout des jumeaux. En 2009 le pourcentage de jumeaux après PMA était de $23,1 \%$, contre 5,7\% seulement en Suède. Les grossesses gémellaires sont hypothéquées par un pourcentage significativement plus élevé de complications, comprenant prématurité et handicaps la vie durant. Ces complications engendrent des coûts importants pour le système de santé qui pourraient être prévenus par le transfert d'un seul embryon, choisi parmi une cohorte d'embryons si les autres pouvaient être congelés. La législation interdit cette pratique en Suisse actuellement. L'intention initiale du législateur de limiter le nombre d'embryons transférés à 3 visait à diminuer les grossesses multiples. Cette règle est maintenant dépassée et doit d'urgence être révisée.

Cet élément est également mis en évidence par l'impossibilité de mettre en œuvre le diagnostic préimplantatoire pourtant approuvé par la majorité du Parlement. Cette décision ne peut être mise en pratique avec la législation actuelle sur la PMA. Nos autorités l'ont reconnu et ont décidé de modifier la loi dans le cadre du diagnostic préimplantatoire. Limiter les changements à ce domaine ajouterait à la confusion actuelle et ne peut se justifier rationnellement.

Enfin, alors que le don de sperme est admis, son pendant féminin, le don d'ovocytes est interdit. Nombre de jeunes femmes, affectées par un cancer sont actuellement efficacement soignées par des traitements anticancéreux (chimiothérapie et radiothérapie) au dépens de leur fertilité. Après leur guérison, ces femmes ne peuvent devenir enceintes qu'à l'aide d'un don d'ovocyte. D'autres femmes, trop âgées pour produire leurs propres ovocytes viables, se font traiter à l'étranger, comme le suggèrent les statistiques. Le nombre de femmes accouchant après 45 ans a été multiplié par 4 depuis 2001. La Cour Européenne de Justice a décidé récemment que les restrictions légales en vigueur en Autriche, similaires aux nôtres, étaient discriminatoires et que le don d'ovocytes devait être autorisé par analogie au don de sperme. Dans le but d'éviter un tourisme médical incontrôlé, une adaptation légale est nécessaire.

Nous demandons une révision des restrictions en cours actuellement. Les modifications minimales suivantes sont demandées: 
1. Pas de restriction du nombre d'ovocytes fertilisés et développés in vitro jusqu'au stade embryonnaire $\left(5^{\mathrm{e}} \mathrm{j}\right.$. environ)

2. Abandon de l'interdiction de congeler des embryons jusqu'au stade d'implantation potentielle (blastocyste)

3. Permettre le don d'ovocytes en suivant des règles semblables à celles qui régissent le don de sperme dans les limites d'indication médicales, comme la perte prématurée de la fonction ovarienne due à une maladie, à un traitement anticancéreux ou encore pour prévenir la transmission de maladies génétiques graves.
A l'exemple d'autre pays, une limitation du nombre d'embryons transférés devrait être encouragée pour prévenir les grossesses multiples. Cette stratégie ne peut être mise en œuvre que si la société est prête à soutenir financièrement les couples.

Approuvé à l'unanimité par le comité de la SSMR le 31.3.11,

Dr Gabriel de Candolle, Prof. Dr Christian De Geyter

\section{Connaissez vous ...}

... les deux revues médicales spécialisées les plus lues en Suisse? Vous tenez l'une d'entre elles entre vos mains.

Le "Bulletin des médecins suisses» et «Forum Médical Suisse» sont les deux revues médicales spécialisées les plus lues des médecins suisses.

Nous vous remercions de votre attention et de votre fidélité. Bonne lecture!

A votre service, les Editions médicales suisses EMH, la maison d'édition des médecins suisses.

Vous trouverez d'autres informations sur le site www.emh.ch.
Taux de lecture le plus élevé (TLE) à l'échelle suisse, tous les médecins, $\mathbf{n}=\mathbf{2 8 0 0}$

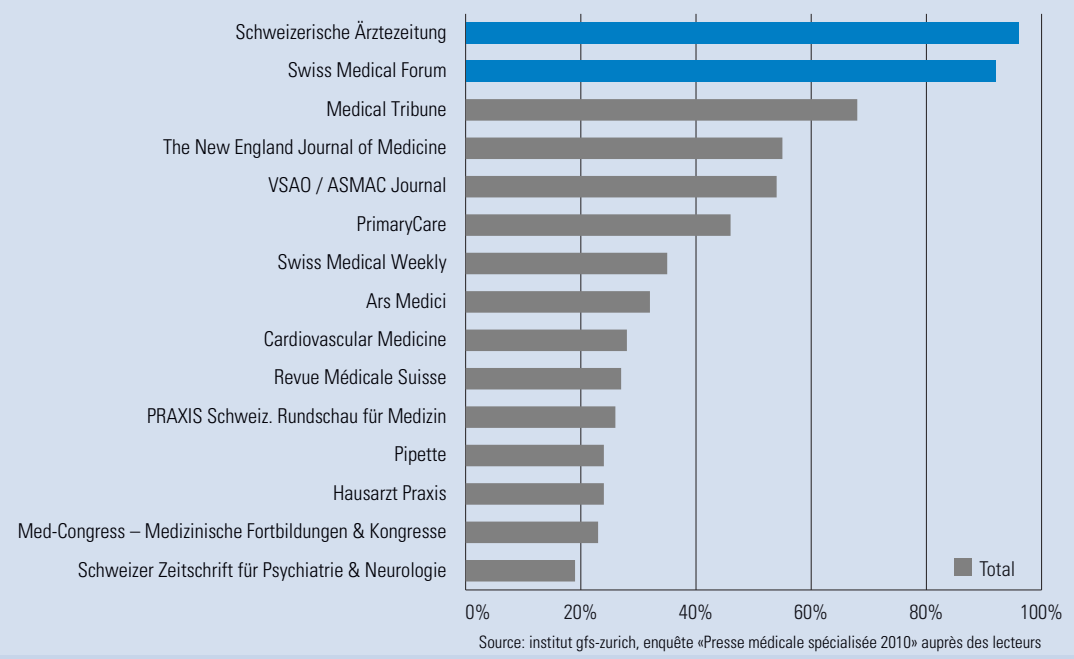

